\title{
Strawberry Dioxygenase: Vorapaxar A model for Antiplatelet Therapy
}

\author{
Alok Jha ${ }^{1}$, Ramasare Prasad ${ }^{2}$ \\ ${ }^{1}$ (Department of Biotechnology, Indian Institute of Technology Roorkee, India) \\ 2 (Department of Biotechnology, Indian Institute of Technology Roorkee, India)
}

\begin{abstract}
A dioxygenase is an enzyme which incorporates both the atoms of molecular oxygen into kinds of substrate, undergoing various types of mechanisms. One of the most important functions of dioxygenases could be the cleavage of aromatic ring compounds, which play key roles in the degradation of aromatic compounds. Tissue factor (TF) is the membrane receptor that initiates coagulation pathway and it promotes developmental and tumor angiogenesis. The gut microbiota promotes TF-glycosylation and activation of coagulation protease. The anti-TF therapy reduces microbiota induced vascular remodeling and expression of the proangiogenic factor angiopoietin 1 (Ang-1) in the small intestine. Here, we report the Protease activated receptor PAR-1 antagonist Vorapaxar binding with the dioxygenase. The structure model suggests that how a highly lipophilic substrate can interact with the membrane integrated protein such as dioxygenase. Dioxygenase can be an important target for drug development for combinatorial drug therapy. The structure model reported here will aid the development of improved PAR-1 antagonist and also improve the understanding of Vorapaxar mode of actions and will justify its use for treatments of intracranial hemorrhages, coronary vascularization and peripheral arterial disease (PAD). We anticipate the structure model will also aid to the structure aided drug designing.
\end{abstract}

Keywords: Dioxygenase, Gut microbiota, Protease activated receptor, PAR1, Tissue factor, TF, Vorapaxar

\section{Introduction}

The stereo selective oxidation of an unactivated carbon-hydrogen bond and the functional group intercoversions, such reactions are mostly carried out by metal dependent oxygenases or oxidases in nature. The best characterized of these enzymes are the cytochrome P450 monoxygenases, ${ }^{(1-3)}$ which belong to the oxygenases or oxidases that use cofactor iron and constitute a 'superfamily' of redox enzymes, the group extends to both diiron-using enzymes for example, methane monoxygenases and ribonucleotide reductase and monoiron-using enzymes that includes those dependent on Fe(III), e.g. Lipoxygenase and the intradiol cleaving catechol dioxygenases and those dependent on $\mathrm{Fe}(\mathrm{II})$, for example, the extradiol cleaving catechol dioxygenases, tyrosine/phenylalanine hydroxylases, benzene/naphthalene dioxygenases and the 2-oxoglutarate (2OG)-dependent dioxygenases. ${ }^{(4)}$ A dioxygenase is an enzyme which incorporates both the atoms of molecular oxygen into kinds of substrates. Oxidative reactions catalyzed by 2OG-dependent dioxygenases are mechanistic steps in the biosynthesis of a variety of metabolites in plants as well as in mammals including flavor compounds and materials of medicinal and agrochemical importance, such as plant hormones' (e.g. gibberellins, abscisic acid) and antibiotics (cephalosporin and the $\beta$-lactamase inhibitor clavulanic acid).

In mammals the gut microbiota is a complex ecosystem coevolved with the host physiology. Various studies in recent times showed gut microbiota interactions with several signaling pathways in which involve essentially dioxygenase functions. One of such study showed that colonization of germ free (GM) mice with a microbiota promotes increased vessel density in the small intestine ${ }^{(5)}$, however, the mechanism involved is little known. Tissue factor (TF) is the membrane receptor that initiates the coagulation pathway ${ }^{(6)}$ and it promotes developmental and tumor angiogenesis. ${ }^{(7,8)}$ The gut microbiota promotes TF glycosylation associated with localization of TF on cell surface, the activation of coagulation protease and phosphorylation of the TF cytoplasmic domain in the small intestine. The study implicated anti-TF treatment decreases microbiota induced vascular remodeling and expression of the proangiogenic factor angiopoietin-1 (Ang-1) in the small intestine. Coagulation protease downstream of TF activates protease activated receptor (PAR) signaling implicated in angiogenesis. ${ }^{(9)}$ The study also showed that PAR-1 plays important role in vessel density and in the 
phosphorylation of cytoplasmic domain of TF, while inhibition of thrombin showed that thrombin-PAR1 signaling is upstream of TF phosphorylation. Thus, the microbiota-induced extra vascular TF-PAR1 signaling loop is a novel pathway that can be modulated to influence vascular remodeling in the small intestine. ${ }^{(\mathbf{1 0})}$ The crystal structure of PAR-1 ${ }^{(11)}$ demonstrated the $2.2 \mathrm{~A}^{\circ}$ resolution crystal structure of human protease activated receptor 1 (PAR1) bound to vorapaxar, a PAR1 antagonist. In adult mammals, the four members of PAR family link tissue injury and local generation of active coagulation proteases to cellular responses that help to regulate haemostasis, thrombosis, inflammation and tissue repair. ${ }^{(13,20)}$ PARs also participate in progression of specific types of cancers. ${ }^{(21,22)}$ In contrast to typical receptor-agonist binding interaction, the interaction of PAR-1 with its activator, thrombin, is that of a protease substrate and dynamic as thrombin transiently binds to the receptor, cleaves it and dissociates. ${ }^{(12,14-18,23)}$ Studies indicate that PAR-1 signaling must be actively terminated ${ }^{(24,26)}$ and unlike most other GPCRs, that can go many rounds of activation by reversible diffusible hormones and neurotransmitters, PARs are degraded after a single activation. ${ }^{(17,19,24-28)}$ As we know, angiogenesis is the formation of new blood vessels and this process of new vessel formation is very important during embryonic development and in the development of diseases, blood vessel network form and expand by sprouting, similar to the way trees grow new branches. This process allows fresh oxygen and nutrients to be delivered to tissues, whether in the developing embryo or tumors. Blood vessel formation and blood vessel damage both are spurred by a variety of chemical signals, which involve complex pathways mediated by various receptors, proteins, enzymes, effectors, natural inhibitors and activators. Dioxygenase is also an important nodal point in these signaling pathways leading to angiogenesis, tumorigenesis, cell differentiation, immune responses and cell survival. The RPE65 superfamily in vertebrates and other 9-cis-epoxycarotenoid dioxygenases (NCED) are involved in retinoid biosynthesis in retinal tissues and carotenoid biosynthesis and metabolism. Other $\alpha-$ ketoglutarate dependent enzymes such as plant and bacterial 4-hydroxyphenylpyruvate dioxygenase and carotenoid cleavage dioxygenases (CCDs) such as maize VP14 are some membrane bound proteins that share common metal coordination as suggested by their crystal structures. ${ }^{(30,31)}$ Such active site coordination is also found in Fumarate reductase and Photosystem II enzymes and in humans with a slight difference in Kynurenine pathway enzymes Tryptophan-2,3-dioxygenase and Indoleamine-2,3-dioxygenase, ${ }^{(32)}$ that are involved in Tryptophan catabolism. Interestingly, these enzymes and RPE65 relatives are membrane bound whereas $\beta$ carotene dioxygenases are water soluble proteins. Substrate for 9-cisepoxy dioxygenase are hydrophobic carotenoids that have essentially no water solubility and because the active sites are located deep within the carotenoid oxygenases rather than near their surfaces, the substrate must be physically removed from the membrane for enzymatic processing. Delivery of substrates in micelles or bilayer is required for their enzymatic processing by these enzymes, thus, it is likely that the enzyme regardless of the degree of their water solubility, must interact with micelles or bilayers to extract substrates.

To elucidate molecular mechanism and function of dioxygenases, we launched a systematic effort to explore the dietary potential of strawberry as a diet supplement and the strawberry dioxygenase as a module for linking plant and human dioxygenase functions in oxidative stress and other similar conditions such as vascular remodeling and cell proliferation or tumorigenesis. Since, dioxygenases have been the targets of rigorous structural investigations, but none of the study combines dioxygenases with the complex processes such as angiogenesis and vascular remodeling. Here, we report the dioxygenase structure model bound to PAR-1 antagonist Vorapaxar, that can shed new lights on the role of dioxygenases in vascular remodeling and the study can provide useful structural insights to further improve the efficiency of Vorapaxar for treating coronary vascularization and severe bleeding problems. This study can also help enhance chances of Vorapaxar to be used for treatments of intracranial hemorrhages and peripheral arterial disease (PAD).

\section{Strawberry Dioxygenase: Overall structure}




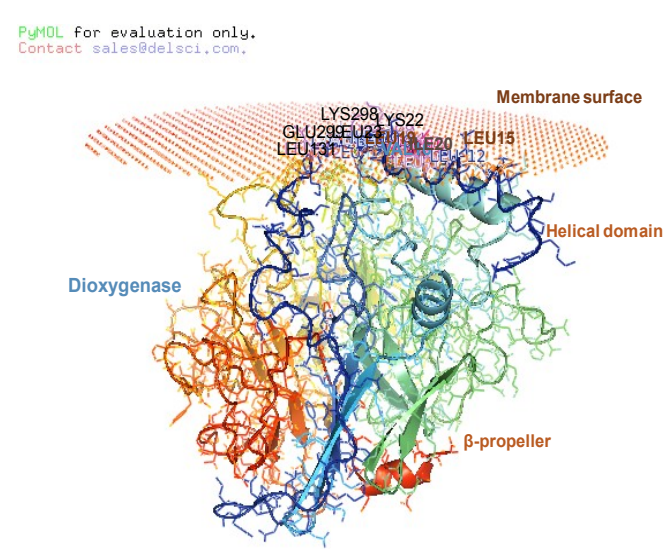

Fig1. dioxygenase membrane orientation

The dioxygenase contains various folds and transmembrane segments with amino and carboxy termini both located on the intracellular side. ${ }^{(29)}$ The transmembrane segments are organized into two distinct domains the $\mathrm{N}$ and $\mathrm{C}$ domains. The transmembrane segments consist of both $\alpha$-helical domain and $\beta$-sheets. The $\alpha$-helical domain seems to be interacting with the membrane surface, while $\beta$-strands with medium size loops are discontinuous which can facilitate conformational changes during substrate transport as dioxygenases belong to the group of enzymes where substrate binding is a dynamic process which means, the substrates are channelized after binding to the dioxygenase enzyme for further processes. The overall structure of dioxygenase is an outward facing conformation and the substrates are likely to be trapped within the centre of the transmembrane domain completely or minimally occluded from the intracellular side yet solvent accessible through the extracellular side through a channel or pore which is of $8-40 \mathrm{~A}^{\circ}$ diameter and presumably not too wide for the substrate to escape. The structure model of dioxygenase with and without substrate may represent an important expansion to the collection of dioxygenase conformations.

\subsection{Structural insights: Dioxygenase and Vorapaxar binding}

Vorapaxar binds in an unusual location in the dioxygenase structure that is very close to the extracellular surface by contrast other substrates penetrate more deeply into the transmembrane core. The Vorapaxar binding pocket, composed of residues from various loops present in the structure including $\alpha$ helices and $\beta$-strands forms a tunnel across the structure with one end open between the loop and $\beta$-sheet $B$ and the other between another loop and $\beta$-sheet $\mathrm{H}$ occupied by the ethyl carbamate tail of Vorapaxar. There is only a small opening in the extracellular surface between the loop and $\beta$-sheet $B$, which leads to the cavity surrounded by $\beta$-sheets $\mathrm{H}$ and $\mathrm{B}$. The base of Vorapaxar binding pocket is composed of $\alpha$ helical domain and $\gamma$-turn residues.

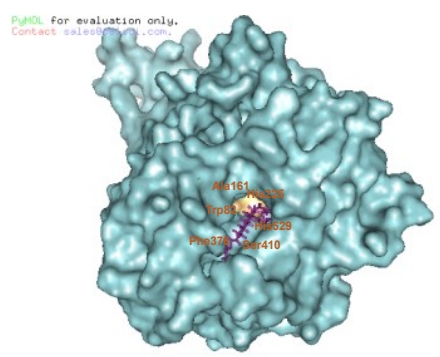

Fig2. dioxygenase-vorapaxar

\subsection{Vorapaxar binding pocket:}

The surface architecture of the Vorapaxar binding pocket shows rigid as well as some flexibility in the structure conformations as illustrated in "Fig 3". 

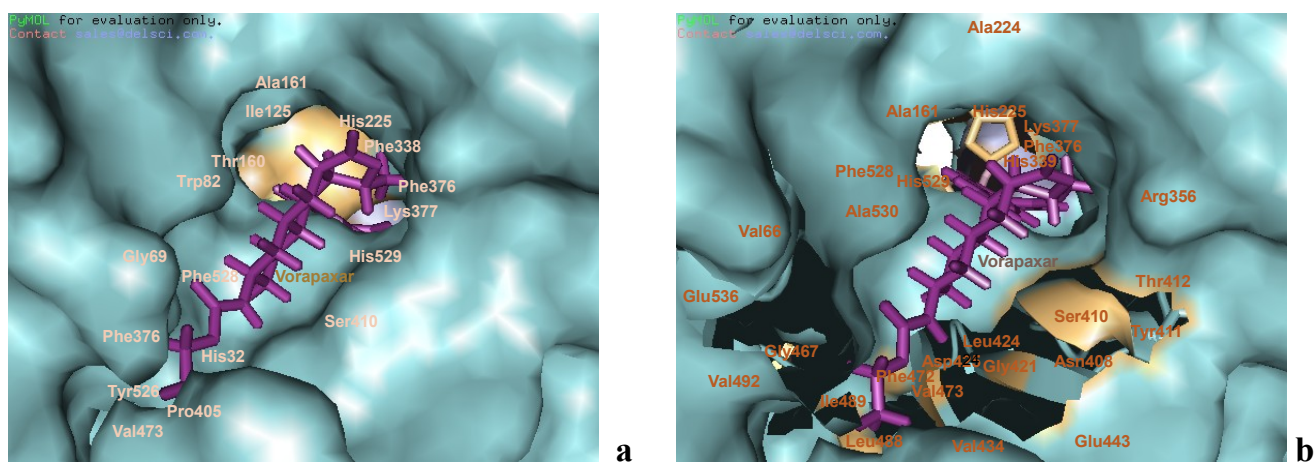

Fig.3 vorapaxar binding pocket: a. His 225 , His339, Ala161 form the open end of the binding pocket whereas Phe376, Pro405 and Val473 contribute to the formation of closed end, Trp82 and Gly69 provide rigid conformations to the vorapaxar binding pocket. b. Some flexible residues Phe472, Asp425, Tyr411, Thr412 and

Ser410, along with Ala530, Val492 and Glu536 form cage like structure which surrounds vorapaxar in the binding pocket. Phe528, His32 and Tyr526 are forming the base of vorapaxar binding pocket.

The closed end of the vorapaxar binding pocket involves residues such as Pro405, Val473, Phe376 and Tyr526 which provide some rigidity, other region in the binding pocket that involves Thr160 and Trp82 including Gly69 is hydrophobic and rather rigid than the region which involves some flexible residues including Gly421, Leu424, Phe472, Asn408, Ser410 and Tyr411 and the residues such as Leu438 and Ile489 can form weak hydrophobic interactions with ethyl carbamate tail of Vorapaxar. The open end of the Vorapaxar which leads to another cavity interior to the structure involves some polar residues that may be interacting with the aromatic ring of vorapaxar by vanderwaal forces and also by forming hydrogen bonds.

The Vorapaxar binding pocket is located on the central axis of $\beta$-propeller structure and is composed of $\alpha$-helical domain and long loops. These loops seem to be playing important structural role in holding and accommodating the substrate. The $\alpha$-helices which are participating in the Vorapaxar binding pocket formation includes $\alpha_{2}$ (Pro30-Tyr33), $\alpha_{4}$ (Gly133-Leu146) and $\alpha_{8}$ (Glu535-Glu540) and part of the $\beta$-propeller structure which consists of anti-parallel $\beta$-sheets B,D,G,H and A including $\beta$-strands, $\beta 5$ (Leu162-His165) with an edge, while $\beta 10$ (Val247-Ser253) is without edge, and other $\beta$-strands, that all are with edges $\beta 20$ (Val402-Val407), $\beta 24(P h e 466-P r o 474), \beta 25$ (Gly486-His493), $\beta 26$ (Val533-Thr534) respectively. The long loops which hold Vorapaxar in the substrate binding pocket contains many $\beta$-turns, $\gamma$-turns and $\beta$-hairpins including interacting residues. The Vorapaxar bound and unbound dioxygenase structure shows the conformational changes upon Vorapaxar binding and the shifting of amino acid residues in the rearrangement process, while, accommodating the substrate in the substrate binding pocket. This is clear from the superimposed structure of Vorapaxar bound and unbound dioxygenase structure that the most possible variation in the position of amino acid residues in order to accommodating the substrate comes through shifting of residues located on such long loops. Residues such as Tyr420, Val407, Arg406 and Arg67 are some residues that are not superimposed in the substrate binding pocket suggesting possible conformational changes upon Vorapaxar binding.
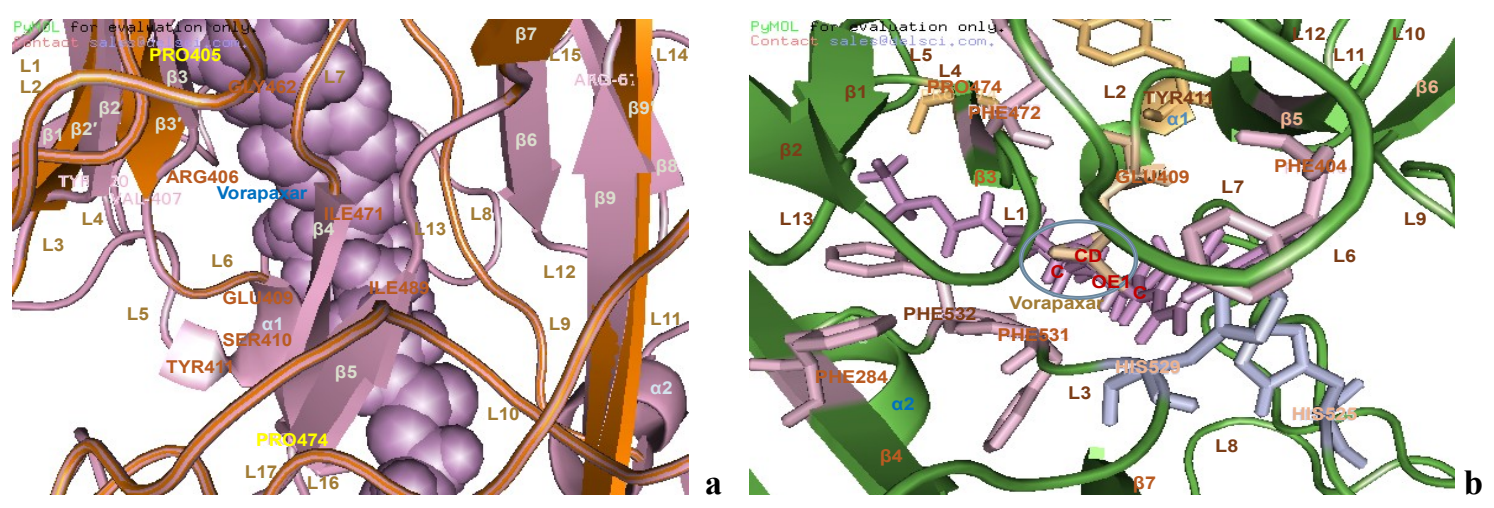

Fig. 4 Conformational changes upon vorapaxar binding. a. The vorapaxar bound-unbound superposition (violetorange) indicates the conformational changes in the overall structure and the substrate binding pocket, Val407, 
Arg406,Tyr420, Arg67 and Pro474 are some residues that shift or undergo conformational changes along with the $\alpha 1$ residue Ser410 upon vorapaxar binding, these changes are mediated by long loops present in the structure. b. Glu409 on loop L2 in the picture binds to the vorapaxar carbon atom by its CD and OE1 atoms and His529 on loop L3 in the picture binds to the carbon atom of vorapaxar by its ND1 atom. The loop between $\beta$ strands $\beta 1, \beta 2$ and $\beta 4$ contains Phe472, Pro474 and Phe284 which undergo some shifting in the rearrangement process to accommodate the vorapaxar in the binding pocket. The loop L3 along with His529 contains Phe531 and Phe532 which interact with the vorapaxar aliphatic chain or probably pyridine ring. $\alpha 1$ residue Tyr411 gets phosphorylated by INSR as the helix is exposed to the kinases due to conformational changes upon vorapaxar binding.

The structural basis of Vorapaxar binding to the dioxygenase is apparent from the dioxygenaseVorapaxar complex structure model and nearly all the residues that interact with Vorapaxar are presumably conserved in all dioxygenase types. The $\beta$-turns which comprise the Vorapaxar binding site contain various interacting residues that are interacting with Vorapaxar and possibly some of them also form hydrogen bonds. The long loops involved in forming the Vorapaxar binding pocket also contains $7 \gamma$-turns, $\gamma 5$ (Thr223-His225), $\gamma 7$ (Ile270-Met272), $\gamma 8$ (Pro288-Tyr290), $\gamma 18$ (Gly367-Ile369) and $\gamma 19$ (Asp374-Phe376), $\gamma 20$ (Thr375Lys377), $\gamma 28$ (Pro477-Ile489), which form the closed end of the Vorapaxar binding pocket. The vorapaxar binding pocket is composed of loops that also contain $\beta$-hairpins of lengths 4 residues, BH3 (Leu162-His 165) of class 2:2IP, 7 residues, BH6 (Val247-Ser253) of class 3:3, 6 residues BH12 (Val402-Val407) of class 9:11, and 9 residues BH15 (Phe466-Pro474) of class 9:11. At the open end of Vorapaxar binding pocket Ala161 form a $\beta$ bulge of class antiparallel G1 along with Thr160 and Leu172 at the edge of $\beta$-strand $\beta 5$ (Leu162-His165) and interacts with the aromatic ring of Vorapaxar.

\subsection{Dioxygenase-Vorapaxar interactions:}

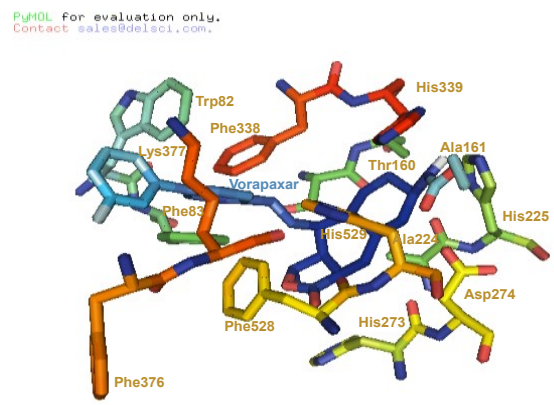

Fig.5. dioxygenase-vorapaxar interacting residues

Residues Glu409 and His529 are forming hydrogen bonds with the aliphatic carbon chain of Vorapaxar, while His529 is interacting with the $6^{\text {th }}$ carbon of Vorapaxar carbamate ring by forming a bond between its nitrogen atom and the hydrogen atom of Vorapaxar carbamate ring. Other active site residues His225 and His529 interact with $\mathrm{N} 1, \mathrm{H} 1, \mathrm{O} 3$ and $\mathrm{O} 4$ atoms by a polar interaction. The residue Asp274 is interacting with Vorapaxar nitrogen and oxygen atoms by a polar interaction, the loop where Asp274 is located also contains a $\gamma$ turn and this $\gamma$ turn (Ile270-Met272) ends with the His273 which is bound to the $\mathrm{Fe}^{2+}$. The $\gamma$-turns $\gamma 5$ (Thr223-His225) and $\gamma 7$ (Ile270-Met272) are structurally important as both the $\gamma$ turns end with catalytic site residues His 225 and His 273 respectively. Phe83 is involved in hydrophobic interactions with carbon atoms along with Ala161 which also interacts with another carbon atom of Vorapaxar. The residue Ala224 on $\gamma$ turn $\gamma 5$ is interacting with initial carbon atoms of Vorapaxar and also along with Phe83, it can provide some substrate selectivity. The active site residues His 225 and His 273 are involved in hydrophobic interactions with other carbon atoms. The residue Phe338 is forming an open end of the Vorapaxar binding pocket along with other binding residues His225 and Phe376. Other active site residues His339 and His529 are also involved in forming a hydrophobic interaction with carbon atoms of Vorapaxar. The oxygen atom of Phe376 is forming a halogen bond with F1 atom of Vorapaxar fluorophenyl ring. The halogen bond interaction with Vorapaxar suggests the inhibitory functions and that chlorinated or fluorinated aromatic compounds as a substrate can be effective inhibitors and useful targets for combinatorial drug therapy. The Vorapaxar halogen atom could be essential for its inhibitory 
functions in cleaving PAR-1. The structure and interaction map of Dioxygenase-Vorapaxar complex indicate that residues Trp82 and Phe83, which contribute to forming the closed end of the Vorapaxar binding pocket are hydrophobic rather rigid. These residues resemble in interaction with the binding mode proposed for Human tryptophan 2, 3-dioxygenase and Indoleamine-2, 3-dioxygenase. Some residues including Trp82, Phe83, His339 and Phe338 at the open end of Vorapaxar binding pocket form $\pi-\pi$ interactions with carbon atoms of Vorapaxar ring. Moreover, the catalytic site residues His225 and His529 are involved in forming cation- $\pi$ interaction with the hydrogen atom. The ammonium group of lysine residue Lys377 can be neutralized by carboxylate and phosphates participate in the anchoring of Vorapaxar carbamate ring at the open end of Vorapaxar binding pocket along with residues Phe376, Phe338, His225 and His339. These interactions may influence ligand binding selectivity indirectly by contributing to the overall structure and stability of the binding pocket. Amino acid residues more distant from the substrate binding pocket may also contribute to the subtype-specific binding of Vorapaxar. However, based on interacting residues, we are able to predict the mechanism by which Vorapaxar gain access to the substrate binding pocket. Although, the structure model is compatible to indicate the slow dissociation rate of Vorapaxar and also the structure does not provide the insight into the actual mechanism of Vorapaxar access to the binding pocket as none of the opening of Vorapaxar binding pocket is large enough to accommodate the passage of ligand, however, the open end of the Vorapaxar binding pocket leads to another cavity or tunnel within the structure as suggested by the pore analysis of the dioxygenase, that the dioxygenase structure contains a tunnel running throughout the structure with a diameter of 3-40 $\mathrm{A}^{\circ}$. To investigate this issue, we analyzed other possible interactions. Moreover, it is interesting to speculate that Vorapaxar a highly lipophilic compound may access the binding pocket through the dioxygenase membrane interacting surface and the $\alpha$-helical domain presumably functions as gate controlling the Vorapaxar access to the active site, the active site is positioned deep within the tunnel through the $\beta$-propeller structure. This is similar to the binding mode proposed for other enzymes of the family such as maize VP14, HPPD and retinal to rhodopsin in the RPE65. ${ }^{(30,31,33)}$ Aromatic amino acid residues Tyr526, Phe528 and His32 form strong interactions with Vorapaxar and residues such as Tyr526, Phe528 and Phe472 assume substantially different positions and linked to each other by hydrogen bonds and form the base of the binding tunnel and are part of a hydrophobic cage that surrounds the ligand. Intriguingly, the incorporation of Vorapaxar in the binding pocket can lead to shifting of some flexible residues such as Phe472 and four proline residues Pro178, Pro230, Pro474 and Pro477 at four corners of the Vorapaxar binding pocket can shift a little or distally to accommodate the substrate while the conformational changes in Vorapaxar bound and unbound stages indicate that removal of Vorapaxar from the binding pocket will not lead to a more open binding pocket but instead to one that is even more closed and also the Vorapaxar binding energy suggested that both Vorapaxar and the substrate binding pocket is less charged and removal of Vorapaxar can lead to collapse of the substrate binding pocket, that can be further investigated by long time-scale dynamic molecular simulation. However, charged residues such as Asp274 can help keeping the substrate binding pocket hydrated as in other receptors.
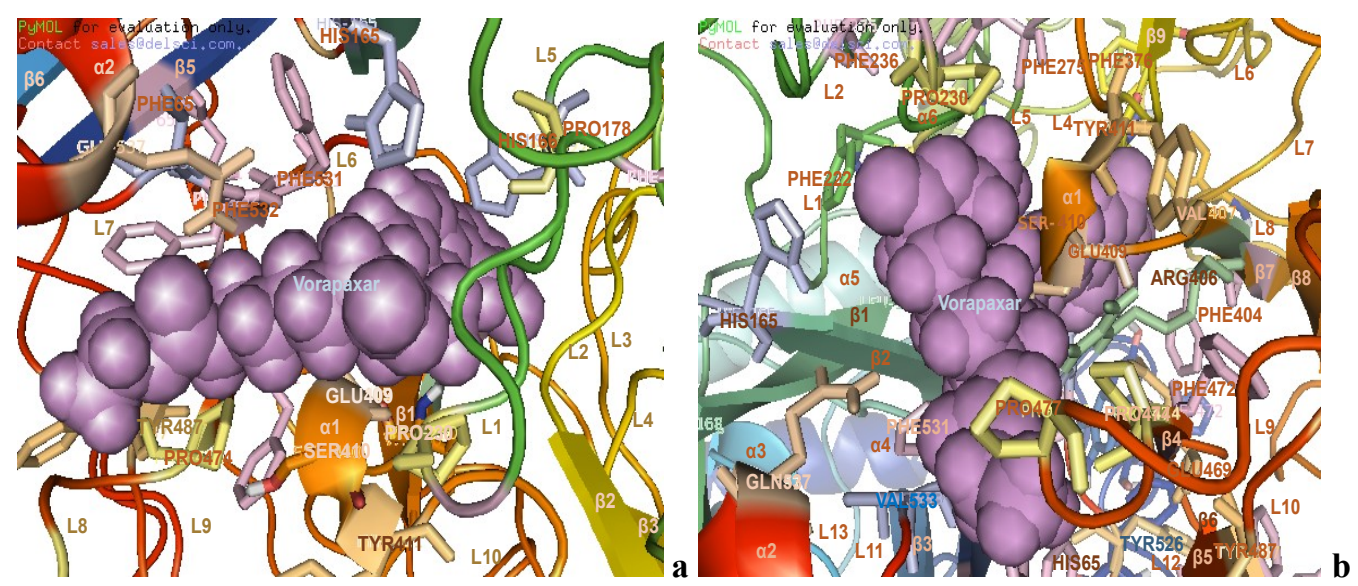

Fig.6. vorapaxar binding pocket conformations. 
The mechanism of Vorapaxar binding involves three ionizing groups in the substrate binding pocket as depicted in "Fig.6"; 2,3 His residues His165, His166 besides active site His residues His225, His529 and His339, and Glu409 and Asp274; the nucleophilic attack and activation of Vorapaxar aromatic ring by His225, His529 and the final deprotonation at His339. The residues Phe531, Phe532, Phe65 on loop L6 participate in the electron charge transfer along with His165, His166 and $\alpha$ helix $\alpha 1$ residue Ser410, Tyr411, Thr412 and Glu409 in the picture, in this process which results in the deamidation of Thr 412 and Gln 537 located on $\alpha$-helix $\alpha 2$, amination or endogenous $\mathrm{CO}_{2}$ production from the carbonyl carbon of Glu409 is also possible, which can lower the $\mathrm{pH}$ in the substrate binding pocket. Asn 408 can also be glycosylated while Thr412 and Tyr411 are phosphorylated by INSR as the Vorapaxar binding exposes the helix $\alpha 1$ to multiple kinases. The Proline residues at four corners of Vorapaxar binding pocket Pro178, Pro230, Pro474 and Pro477 undergo shifting to accommodate the ligand.

The structure model seem to agree on $\alpha$-ketoacid moiety binding through bidentate coordination with the metal ion and the hydrogen atom of conserved residues such as glutamine, Gln537 and glutamate, Glu409; but to explain the binding of hydroxyl group, other approaches are taken into consideration, however, the substrate Vorapaxar itself explains the binding of hydroxyl group, that the hydroxyl group at such positions lead to the hydroxylation of corresponding residues, thus Glu409 is hydroxylated. The structure complex contains three different ionizable groups, such as His165, His166 and catalytic site residues His225, His339 and His529 along with Glu409 and Asp274 are interacting with the substrate that satisfies the rules of His ${ }^{1}$-Glu/Asp-His ${ }^{2}$, here Glu409 binds the substrate facilitating electron transfer and the nucleophilic attack and activation of the substrate aromatic ring by His 225 and His529 and subsequent deprotonation step at His339. The binding of Glu409 with Vorapaxar causes the $\alpha$-helix $\alpha 7$ in the structure to get strained and the kink in the $\alpha$ helix due to Ser410 is exposed to protein kinase C (PKC), thus Ser410 is phosphorylated, other residue on the same $\alpha$-helix Tyr411 and Thr412 are also phosphorylated by INSR and protein kinase C (PKC) respectively. Tyr411 can also be hydroxylated and the hydroxyl groups of both Tyr411 and Thr412 form hydrogen bond with each other or with the Vorapaxar ring carbon, whereas, Lys377 provides stability to the structure complex and is a potential target for acetylation. Other interacting residue are also phosphorylated such as Thr160 is phosphorylated by cdc2, Ser 481 by CKII, Thr497 by PKC, Thr534 by CKII and Tyr420 by INSR. Tyr 116 is phosphorylated by SRC while INSR phosphorylates Tyr164 and Tyr197. Tryptophan residues at positions Trp82, Trp329 and Trp344 are supposed to be C-glycosylated; Asparagines residues at positions Asn37 and Asn408 are Nglycosylated while the oxygen atom of Thr265 is O-glycosylated. Ser398 is phosphorylated by cAMP and cGMP dependent protein kinase, Tyr153 is phosphorylated by Tyrosine kinase, Gly156 is N-myristoylated, Thr375 is phosphorylated by protein kinase C (PKC), Ser 173 is phosphorylated by CKII, while Ala440, Ala441, Pro442, Glu443, Val445, Gly446, Lys447, The448 is a ATP-GTP binding site. These are some important functional modifications, which are thought to be correlated with the dioxygenase functions and may be crucial for Vorapaxar signaling pathway in coordination with dioxygenase and multiple kinases in the prevention of clinical disorders. The conventional and dual antiplatelet therapy by combining aspirin with antiplatelet drug clopidogrel or aspirin alone or a combination of aspirin and dipyridamole have been shown to reduce stroke risk in patients with heart disease or prior stroke, but the combination carries a greater risk of gastrointestinal bleeding. Researchers continue to investigate whether Paclitaxel and Vorapaxar (protease activated receptor, PAR1 inhibitor) combination indicated some reduction in major bleeding problems, and the crucial functions of dioxygenase in the VEGF signaling pathway, which is essential for angiogenesis have made dioxygenase one of more challenging enzymes to characterize and a potential target for drug development. The structure model of dioxygenase offers insights into a very high affinity interaction with the PAR-1 antagonist Vorapaxar. The regulation of TNF- $\alpha$ and T-effector cells is mediated by dioxygenase and certain interleukins such as IL-3, IL10, IL-13, IL-17 and IL-27, that are involved in the regulation of regulatory T-cells $\left(\mathrm{T}_{\text {reg }}\right)$, that act as an important brake on inflammation. ${ }^{(34)}$ This could also be implicated in vasculitis and other inflammation in the patients with diabetes type 2 and obesity. The structure complex could provide essential information for the Vorapaxar mode of actions as in terms of PAR-1 inhibition by activating the receptor to be cleaved by thrombin, its natural activator, may interact with dioxygenase which can mediate oxidative transformations leading to the PAR-1 activation or thrombin accumulation. We suggest a combinatorial drug therapy, the combination of PAR-1 inhibitor Vorapaxar with angiotensin converting enzyme (ACE) inhibitor, $\beta$-blocker and angiotensin II receptor blocker drugs, for treating intracranial hemorrhages, coronary vascularization and peripheral arterial disease (PAD). 


\section{Conclusion}

The activation and improved pharmacological and bioinformatics tools helped to investigate dioxygenase functions in signaling pathways that lead to angiogenesis and aortic wall inflammation or vasculitis. The strawberry dioxygenase and vorapaxar complexes had been studied by homology modeling approach and the interacting residues could explain the vorapaxar mode of actions in terms of PAR-1 inhibition by activating the receptor to be cleaved by thrombin, its natural activator, which presumably interacts with dioxygenase which can mediate oxidative transformations leading to the PAR-1 activation or thrombin accumulation. However, the mechanism of vorapaxar access to the active site is poorly understood but the location of amino acids known to influence Vorapaxar suggests that the activation of dioxygenase and the Vorapaxar mechanism of action may have superficial interactions with the long loops present in the dioxygenase structure. Thus, the significance of long loops in the protein structure and their role in holding the substrate in the substrate binding pocket and the role of tyrosine phosphorylated enzymes such as dioxygenase provide required information about enzymatic activity under stress or similar conditions.

\section{Methods Summary}

We analyzed the protein sequence before generating the structural model as there are certain specificities required for validating the concept of homology modeling. Any protein sequence must be analyzed as it may provide necessary information, based on which one can study the protein. Therefore, we deduced the strawberry dioxygenase amino acid sequence from NCBI database (www.ncbi.gov.in). We analyzed the sequence for $\beta$-barrel outer membrane protein by using PRED-TMBB server. (biophysics.biol.uoa.gr/PREDTMBB/). To confirm the membrane orientation of dioxygenase, we used PPM server, (opm.phar.umich.edu/server.php) that indicated the tilt of dioxygenase protein from the membrane and membrane buried residues. The dioxygenase sequence was analyzed for secondary structure and topology predictions, using PSI-PRED (128.16.10.201/psipred/) and EMBL-EBI (www.ebi.ac.uk/) tool ProFunc for protein functions. With more than $30 \%$ similarity and less gap in alignment, the template 3NPE_A served as an ideal template for generating dioxygenase structure. We generated the dioxygenase protein structure using Geno3D server. (geno3d-pbil.ibcp.fr/). The models were generated with the multipass model expectation value threshold 0.002 in the expectation value of 10 and the alignment was done in the matrix BLOSUM62. We deduced 3D structure of substrate vorapaxar (CID10077130) from PubChem compound (www.ncbi.nlm.nih.gov). The substrate docking studies were performed by using Hex (hex.loria.fr/), and dockingserver (www.dockingserver.com/). The post translational modifications such as phosphorylation, glycosylation, amidation, acetylation studies were performed by using MyHits (myhits.isb-sib.ch/cgibin/motif_scan) and LRR finder (www.Irrfinder.com/) servers. The pictures were generated by PDB viewer PyMol (www.pymol.org/).

\section{References}

[1]. Wong LL: Cytochrome P450 monoxygenases. Curr Opin Chem Biol 1998; 2:263-268.

[2]. Ortz de Montellano PR: Cytochrome P450: Structure, Mechanisms and Biochemistry, edn 2. London: Plenum Press; 1995.

[3]. Lange SJ, Que L: Oxygen activating nonheme iron enzymes. Curr Opin Chem Biol 1998; 2:159-172.

[4]. Schofield CJ and Zhang Zhihong: Structural and mechanistic studies on 2-oxoglutarate-dependent oxygenases and related enzymes. Curr Opin Struct Biol 1999; 9:722-731.

[5]. Stappenbeck, T. S., Hooper, L. V. \& Gordon, J. I. Developmental regulation of intestinal angiogenesis by indigenous microbes via Paneth cells. Proc. Natl Acad. Sci. 2002; USA 99: 15451-15455.

[6]. Morrissey, J. H., Fakhrai, H. \& Edgington, T. S. Molecular cloning of the cDNA for tissue factor, the cellular receptor for the initiation of the coagulation protease cascade. Cell 1987; 50:129-135.

[7]. Carmeliet, P. et al. Role of tissue factor in embryonic blood vessel development. Nature. 1996; 383:73-75.

[8]. Belting, M. et al. Regulation of angiogenesis by tissue factor cytoplasmic domain signaling. Nature Med. $2004 ; 10: 502-509$.

[9]. Griffin, T. C., Srinivasan, Y., Zheng, Y.-W., Huang, W. \& Coughlin, S. R. A role for thrombin receptor signaling in endothelial cells during embryonic development. Science. 2001; 293:1666-1670.

[10]. Reinhardt, Christoph., Bergentall, Mattias., Greiner,Thomas U., Schaffner, Florence, Lunde'n, GunnelO“ stergren. Petersen, Lars C., Ruf. Wolfram., Ba“ckhed, Fredrik., Tissue factor and PAR1 promote microbiota-induced intestinal vascular remodeling. Nature. 2012; 483:627-631. 
[11]. Zhang, Cheng., Srinivasan,Yoga., Arlow, Daniel H., Fung, Juan Jose.,Palmer, Daniel., Zheng, Yaowu., Green, Hillary F., Pandey, Anjali., Dror, Ron O., Shaw, David E., Weis, William I., Coughlin, Shaun R., K. Kobilka, Brian. High-resolution crystal structure of human protease-activated receptor 1. Nature. 2012; 492:387-392.

[12]. Vu, T. K., Hung, D. T., Wheaton, V. I. \& Coughlin, S. R. Molecular cloning of a functional thrombin receptor reveals a novel proteolytic mechanism of receptor activation. Cell. 1991; 64:1057-1068.

[13]. Coughlin, S. R. Thrombin signalling and protease-activated receptors. Nature 2000; 407: $258-264$.

[14]. Vu, T. K., Wheaton, V. I., Hung, D. T., Charo, I. \& Coughlin, S. R. Domains specifying thrombin-receptor interaction. Nature. 1991; 353: 674-677.

[15]. Chen, J., Ishii, M., Wang, L., Ishii, K. \& Coughlin, S. R. Thrombin receptor activation. Confirmation of the intramolecular tethered liganding hypothesis and discovery of an alternative intermolecular liganding mode. J. Biol. Chem. 1994; 269:16041-16045.

[16]. Liu, L. W., Vu, T. K., Esmon, C. T. \& Coughlin, S. R. The region of the thrombin receptor resembling hirudin binds to thrombin and alters enzyme specificity. J. Biol. Chem. 1991; 266: 16977-16980.

[17]. Ishii, K., Hein, L.,Kobilka, B.\&Coughlin, S. R. Kinetics of thrombin receptor cleavage on intact cells. Relation to signaling. J. Biol. Chem. 1993, 268: 9780-9786.

[18]. Mathews, I. I. et al. Crystallographic structures of thrombin complexed with thrombin receptor peptides: existence of expected and novel binding modes. Biochemistry. 1994, 33, 3266-3279.

[19]. Gerszten, R. E. et al. Specificity of the thrombin receptor for agonist peptide is defined by its extracellular surface. Nature. 1994; 368:648-651.

[20]. Coughlin, S. R. Protease-activated receptors in hemostasis, thrombosis and vascular biology. J. Thromb. Haemost. 2005; 3:18001814.

[21]. Garci'a-Lo'pez, M. T., Gutierrez-Rodriguez, M. \& Herranz, R. Thrombin-activated receptors: promising targets for cancer therapy? Curr. Med. Chem. 2010; 17: 109-128.

[22]. Ramachandran, R., Noorbakhsh, F., Defea, K. \& Hollenberg, M. D. Targeting proteinase activated receptors: therapeutic potential and challenges. Nature Rev. Drug Discov. 2012;11: 69-86.

[23]. Ishii, K. et al. Determinants of thrombin receptor cleavage. Receptor domains involved, specificity, and role of the P3 aspartate. $J$. Biol. Chem. 1995; 270:16435-16440.

[24]. Shapiro, M. J., Trejo, J., Zeng, D. \& Coughlin, S. R. Role of the thrombin receptor's cytoplasmic tail in intracellular trafficking. Distinct determinants for agonist triggered versus tonic internalization and intracellular localization. J. Biol. Chem. 1996; 271: 32874-32880.

[25]. Trejo, J. \& Coughlin, S. R. The cytoplasmic tails of protease-activated receptor-1 and substance P receptor specify sorting to lysosomes versus recycling. J. Biol.Chem. 1999; 274: 2216-2224.

[26]. Trejo, J., Hammes, S. R. \& Coughlin, S. R. Termination of signaling by protease activated receptor-1 is linked to lysosomal sorting. Proc. Natl Acad. Sci. 1998; USA 95, 13698-13702.

[27]. Shapiro, M. J. \& Coughlin, S. R. Separate signals for agonist-independent and agonist-triggered trafficking of protease-activated receptor 1. J. Biol. Chem. 1998; 273:29009-29014.

[28]. Hein, L., Ishii, K., Coughlin, S. R. \& Kobilka, B. K. Intracellular targeting and trafficking of thrombin receptors. A novel mechanism for resensitization of a G protein-coupled receptor. J. Biol. Chem. 1994; 269, 27719-27726.

[29]. Ginalski K: Comparative modeling for protein structure prediction. Curr. Opin Struct Biol. 2006; 16:172-177.

[30]. Fritze, Iris M., Linden, Lars., Freigang, Jo“̈rg., Auerbach, Gu“ nter., Huber, Robert., Steinbacher, Stefan., The Crystal Structures of Zea mays and Arabidopsis 4 Hydroxyphenylpyruvate Dioxygenase. Plant Physiology, 2004; 134:1388-1400.

[31]. Messing, Simon A.J., Gabelli, Sandra B., Echeverria, Ignacia., Vogel, Jonathan T., Guan, Jiahn Chou., Tan, Bao Cai., Klee, Harry J.. McCarty, Donald R., Amzela, L. Mario. Structural Insights into Maize Viviparous14, a Key Enzyme in the Biosynthesis of the Phytohormone Abscisic Acid. The Plant Cell, 2010; 22: 2970-2980.

[32]. Zhang, Yang., Kang, Seong A., Mukherjee, Tathagata., Bale, Shridhar., Crane, Brian R. Begley, Tadhg P., Ealick, Steven E. Crystal Structure and Mechanism of Tryptophan 2,3-Dioxygenase, a Heme Enzyme Involved in Tryptophan Catabolism and in Quinolinate Biosynthesis. Biochemistry 2007; 46, 145-155.

[33]. Kiser, Philip D., Golczaka, Marcin., Lodowskia, David T., Chanceb, Mark R., Palczewskia, Krzysztof. Crystal structure of native RPE65, the retinoid isomerase of the visual cycle. PNAS, 2009; 106, 41:17325-17330.

[34]. Montecucco, Fabrizio., Pende, Aldo., Mach, Franc,ois., The Renin-Angiotensin System Modulates Inflammatory Processes in Atherosclerosis: Evidence from Basic Research and Clinical Studies. Mediators of Inflammation Volume 2009, Article ID 752406, 1-13. 2009. 